\title{
A New Parameter Conjugate Gradient Method Based on Three Terms Unconstrained Optimization
}

\author{
Hussein Ageel Khatab ${ }^{1}$ and Salah Gazi Shareef ${ }^{2}$ \\ 1,2 Department of Mathematics, Faculty of Science, University of Zakho, Kurdistan Region, Iraq \\ ${ }^{1}$ husseinkhatab632@gmail.com ,2 salah.gazi2014@gmail.com
}

Abstract. In this paper, we suggest a new conjugate gradient method for solving nonlinear unconstrained optimization problems by using three term conjugate gradient method, We give a descent condition and the sufficient descent condition of the suggested method.

Keywords: Unconstrained Optimization; Conjugate Gradient Method; Descent Condition; Three Term Conjugate Gradient Method.

\section{Introduction}

We consider the unconstrained optimization problem:

$$
\text { Min } f(x), x \in R^{n}
$$

where $f: R^{n} \rightarrow R$ is a real-valued, continuously differentiable function.

A no nlinear conjugate gradient method generates a sequence $\left\{x_{k}\right\}, \mathrm{k} \geq 1$, starting from an initial guess $x_{1} \in R^{n}$, using the recurrence

Or $\quad v_{k}=\alpha_{k} d_{k}$

$$
x_{k+1}=x_{k}+\alpha_{k} d_{k}
$$

Where, $v_{k}=x_{k+1}-x_{k}$

The positive step size $\alpha_{k}$ is obtained by some line search, and $d_{k}$ is a search direction. normally the search direction at the first iteration is the steepest descent direction, namely $d_{1}=-g_{1}$ and the other search directions can be defined as:

$$
d_{k+1}=-g_{k+1}+\beta_{k} d_{k}
$$

Where $g_{k}=\nabla f\left(x_{k}\right)$ and $\beta_{k}$ is a scalar. There are many formulas for $\beta_{k}$, for example, Hestenes-Stiefel (HS)[ 8] , Conjugate descent (CD)[10], Polak-Ribiere-Polyak (PRP)[1] , Dai and Yuan (DY) [11] , (D PRP)[2] , RMIL [9] and $\beta_{k}^{1}$ [12], these formulas are as follows:

where, $t>1 / 4$

$$
\begin{aligned}
\beta_{k}^{H S} & =\frac{g_{k+1}^{T}\left(g_{k+1}-g_{k}\right)}{d_{k}^{T}\left(g_{k+1}-g_{k}\right)} \\
\beta_{k}^{C D} & =\frac{\left\|g_{k+1}\right\|^{2}}{-d_{k}^{T} g_{k}} \\
\beta_{k}^{P R P} & =\frac{g_{k+1}^{T}\left(g_{k+1}-g_{k}\right)}{\left\|g_{k}\right\|^{2}} \\
\beta_{k}^{D Y} & =\frac{\left\|g_{k+1}\right\|^{2}}{d_{k}^{T}\left(g_{k+1}-g_{k}\right)} \\
\beta_{k}^{D P R P} & =\beta_{k}^{R P R}-t \frac{g_{k+1}^{T} d_{k}\left\|y_{k}\right\|^{2}}{\left\|g_{k}\right\|^{4}}
\end{aligned}
$$

$\beta_{k}^{R M I L}=\frac{g_{k+1}^{T}\left(g_{k+1}-g_{k}\right)}{\left\|d_{k}\right\|^{2}}$

where, $t>0$

$$
\beta_{k}^{1}=\frac{g_{k+1}^{T} y_{k}}{d_{k}^{T} y_{k}}-t \frac{g_{k+1}^{T} d_{k}}{\left\|d_{k}\right\|^{2}}
$$


$\mathbf{y}_{\mathbf{k}}=\mathbf{g}_{\mathrm{k}+\mathbf{1}}-\mathbf{g}_{\mathrm{k}}$, symbol $\|$. $\|$ denotes the Euclidean norm of vectors. The most well-studied properties of conjugate gradient methods are its global convergence properties. The convergence of conjugate gradient methods under different line searches has been studied by many authors, such as Gilbert and Nocedal [3]. Al-Baali has proven the descent property and global convergence of the Fletcher-Reeves Method with Inexact Line Search [7].

This paper is organized as follow: in Section 2, we will present a new conjugate gradient method. In Section 3, we prove the descent condition and sufficient descent condition of new method. In Section 4, some numerical experiments to this new conjugate gradient method are presented. In section 5, we will give the conclusion.

\section{New conjugate gradient algorithm}

In this section, we will derive the our suggestion bas ed on a three term conjugate gradi ent method. J. Liu and X. Wu proposed a three-term conjugate gradient method, in which the search direction $d_{k}$ has the form ,

$$
d_{k+1}=-g_{k+1}-\beta_{k} s_{k}+\delta_{k} y_{k}
$$

where $\beta_{k}=\frac{g_{k+1}^{T} s_{k}}{y_{k}^{T} s_{k}}$ and $\delta_{k}=\frac{g_{k+1}^{T} y_{k}}{y_{k}^{T} s_{k}}$, see[4]. Also, There are many three term conjugate gradient algorithm suggested, for instance:

$$
\begin{array}{ll}
d_{k+1}=-g_{k+1}+\beta_{k}^{H S} d_{k}-\frac{g_{k+1}^{T} d_{k}}{d_{k}^{T} y_{k}} y_{k} & \text {, see [6] } \\
d_{k+1}=-g_{k+1}+\beta_{k}^{P R P} d_{k}-\frac{g_{k+1}^{T} d_{k}}{\left\|g_{k}\right\|^{2}} y_{k} & \text {, see[5]. }
\end{array}
$$

We suggest a new three-term conjugate gradient as following:

where, $\delta \in(0,1)$ and $\mu=\frac{g_{k+1}^{T} d_{k}}{\left\|g_{k}\right\|^{2}}$

$$
d_{k+1}=-g_{k+1}+\beta_{k}^{N E W} d_{k}-\delta \mu y_{k}
$$

Now, from equation (2.3) and equation (2.4), we have

$$
\frac{g_{k+1}^{T} y_{k}}{\left\|g_{k}\right\|^{2}} d_{k}-\frac{g_{k+1}^{T} d_{k}}{\left\|g_{k}\right\|^{2}} y_{k}=\beta_{k}^{N E W} d_{k}-\delta \frac{g_{k+1}^{T} d_{k}}{\left\|g_{k}\right\|^{2}} y_{k}
$$

Multiplying both sides of above equation by $d_{k}$, we obtain

This implies that

$$
\frac{g_{k+1}^{T} y_{k}}{\left\|g_{k}\right\|^{2}} d_{k}^{T} d_{k}-\frac{g_{k+1}^{T} d_{k}}{\left\|g_{k}\right\|^{2}} d_{k}^{T} y_{k}=\beta_{k}^{N E W} d_{k}^{T} d_{k}-\delta \frac{g_{k+1}^{T} d_{k}}{\left\|g_{k}\right\|^{2}} d_{k}^{T} y_{k}
$$

So,

$$
\frac{g_{k+1}^{T} y_{k}}{\left\|g_{k}\right\|^{2}}-\frac{g_{k+1}^{T} d_{k} d_{k}^{T} y_{k}}{d_{k}^{T} d_{k}\left\|g_{k}\right\|^{2}}=\beta_{k}^{N E W}-\delta \frac{g_{k+1}^{T} d_{k} d_{k}^{T} y_{k}}{d_{k}^{T} d_{k}\left\|g_{k}\right\|^{2}}
$$

or

$$
\beta_{k}^{N E W}=\frac{g_{k+1}^{T} y_{k}}{\left\|g_{k}\right\|^{2}}+(\delta-1) \frac{g_{k+1}^{T} d_{k} d_{k}^{T} y_{k}}{d_{k}^{T} d_{k}\left\|g_{k}\right\|^{2}}
$$

where , $0<\delta<1$.

$$
\beta_{k}^{N E W}=\beta_{k}^{R P R}+(\delta-1) \frac{g_{k+1}^{T} d_{k} d_{k}^{T} y_{k}}{\left\|d_{k}\right\|^{2}\left\|g_{k}\right\|^{2}}
$$

2.1 Algorithm of a new conjugate gradient $\operatorname{method}\left(\beta_{k}^{N E W}\right)$

Step(1):- Select $x_{1}$ and $\varepsilon=10^{-5}$.

$\operatorname{Step}(2):-\operatorname{Set} d_{1}=-g_{1} \quad, g_{k}=\nabla f\left(x_{k}\right)$, Set $k=1$.

Step(3):- Compute the step length $\alpha_{k}>0$ satisfying the Wolfe line search

$$
\begin{aligned}
& f\left(x_{k}+\alpha_{k} d_{k}\right)-f\left(x_{k}\right) \leq c_{1} \alpha_{k} g_{k}^{T} d_{k} \\
& \left|g_{K+1}^{T} d_{k}\right| \leq c_{2}\left|g_{k}^{T} d_{k}\right|
\end{aligned}
$$

where, $0<c_{1}<c_{2}<1$.

Step(4):- Compute

$$
x_{k+1}=x_{k}+\alpha_{k} d_{k} \text {. }
$$

Step (5):- Compute $\beta_{k}^{N E W}$ by (2.5)

Step (6):- Compute $d_{k+1}=-g_{k+1}+\beta_{k}^{N E W} d_{k}$

Step(7):- If $\left|g_{k+1}^{T} g_{k}\right|>0.2\left\|g_{k+1}\right\|^{2}$ then go to step 2 .

Else 
$k=k+1$ and go to step 3 .

Theorem 2.1:-Assume that the sequence $\left\{x_{k}\right\}$ is generated by (1.2), then the search direction in (1.3) with new conjugate gradient method (2.5) satisfy the descent condition, i.e. $d_{k+1}^{T} g_{k+1} \leq 0$ with exact and inexact line search. Proof:- From (1.3) and (2.5) we have,

$$
d_{k+1}=-g_{k+1}+\left(\frac{g_{k+1}^{T} y_{k}}{\left\|g_{k}\right\|^{2}}+(\delta-1) \frac{g_{k+1}^{T} d_{k} d_{k}^{T} y_{k}}{d_{k}^{T} d_{k}\left\|g_{k}\right\|^{2}}\right) d_{k}
$$

Multiply both sides of above equation by $g_{k+1}$, to obtain

$$
d_{k+1}^{T} g_{k+1}=-\left\|g_{k+1}\right\|^{2}+\frac{g_{k+1}^{T} y_{k}}{\left\|g_{k}\right\|^{2}} d_{k}^{T} g_{k+1}+(\delta-1) \frac{\left(g_{k+1}^{T} d_{k}\right)^{2} d_{k}^{T} y_{k}}{\left\|d_{k}\right\|^{2}\left\|g_{k}\right\|^{2}}
$$

If the step length $\alpha_{k}$ is chosen by an exact line search which requires $d_{k}^{T} g_{k+1}=0$. Then the proof is complete. If the step length $\alpha_{k}$ is chosen by inexact line search which requires $d_{k}^{T} g_{k+1} \neq 0$

the first two terms of equation (2.7) are less than or equal to zero because the param eter of (PRP) is satisfies the descent condition, and the third term clearly is less than or equal to zero, since $d_{k}^{T} y_{k}>0$, so ,

$$
d_{k+1}^{T} g_{k+1}=-\left\|g_{k+1}\right\|^{2}+\frac{g_{k+1}^{T} y_{k}}{\left\|g_{k}\right\|^{2}} d_{k}^{T} g_{k+1}+(\delta-1) \frac{\left(g_{k+1}^{T} d_{k}\right)^{2} d_{k}^{T} y_{k}}{\left\|d_{k}\right\|^{2}\left\|g_{k}\right\|^{2}} \leq 0 .
$$

Theorem 2.2:- :- Assume that the sequence $\left\{x_{k}\right\}$ is generated by (1.2), then the search direction in (1.3) with new conjugate gradient method (2.5) satisfy the sufficient descent condition, i.e.

$$
d_{k+1}^{T} g_{k+1} \leq-C\left\|g_{k+1}\right\|^{2} \text {. }
$$

Proof:- From (1.3) and (2.5) we have

$$
d_{k+1}=-g_{k+1}+\left(\frac{g_{k+1}^{T} y_{k}}{\left\|g_{k}\right\|^{2}}+(\delta-1) \frac{g_{k+1}^{T} d_{k} d_{k}^{T} y_{k}}{d_{k}^{T} d_{k}\left\|g_{k}\right\|^{2}}\right) d_{k}
$$

Multiply both sides of above equation by $g_{k+1}$, to obtain

$$
d_{k+1}^{T} g_{k+1}=-\left\|g_{k+1}\right\|^{2}+\frac{g_{k+1}^{T} y_{k}}{\left\|g_{k}\right\|^{2}} d_{k}^{T} g_{k+1}+(\delta-1) \frac{\left(g_{k+1}^{T} d_{k}\right)^{2} d_{k}^{T} y_{k}}{\left\|d_{k}\right\|^{2}\left\|g_{k}\right\|^{2}}
$$

Now, since the parameter of (PRP) is satisfies the descent condition, then the above equation becomes

$$
\begin{gathered}
d_{k+1}^{T} g_{k+1} \leq(\delta-1) \frac{\left(g_{k+1}^{T} d_{k}\right)^{2} d_{k}^{T} y_{k}}{\left\|d_{k}\right\|^{2}\left\|g_{k}\right\|^{2}} \\
=(\delta-1) \frac{\left(g_{k+1}^{T} d_{k}\right)^{2} d_{k}^{T} y_{k}}{\left\|d_{k}\right\|^{2}\left\|g_{k}\right\|^{2}} * \frac{\left\|g_{k+1}\right\|^{2}}{\left\|g_{k+1}\right\|^{2}} \\
=-\left\|g_{k+1}\right\|^{2}\left[(1-\delta) \frac{\left(g_{k+1}^{T} d_{k}\right)^{2} d_{k}^{T} y_{k}}{\left\|g_{k+1}\right\|^{2}\left\|d_{k}\right\|^{2}\left\|g_{k}\right\|^{2}}\right]
\end{gathered}
$$

Let $C=(1-\delta) \frac{\left(g_{k+1}^{T} d_{k}\right)^{2} d_{k}^{T} y_{k}}{\left\|g_{k+1}\right\|^{2}\left\|d_{k}\right\|^{2}\left\|g_{k}\right\|^{2}}$ which is positive, then

$$
d_{k+1}^{T} g_{k+1} \leq-C\left\|g_{k+1}\right\|^{2} .
$$

\section{Numerical Results}

This section is devoted to test the implementation of new method. We compare the our method with Conjugate Gradient methods ( (PRP), (HS), RMIL and $\beta_{k}^{1}$ ) the comparative tests involve Well-known nonlinear problems (standard test function) with different dimensions $4 \leq n \leq 5000$, all programs are written in FORTRAN 90 language and for all cases the stopping condition is $\left\|g_{k+1}\right\| \leq 10^{-5}$, the results given in table (1) and table (2) specifically quote the number of function NOF and the nuber of iteration NOI . More experimental results in table (1) and table(2) confirm that the new CG is superior to standard CG (PRP), standard CG (HS), RMIL and $\beta_{k}^{1}$ with respect to the NOI and NOF. 
Table (1): Comparative Performance of the Three Algorithms (PRP, HS and New Conjugate Gradient Method)

\begin{tabular}{|c|c|c|c|c|c|c|c|}
\hline \multirow[t]{2}{*}{ Testfunction } & \multirow[t]{2}{*}{$\mathbf{N}$} & \multicolumn{2}{|c|}{ Algorithm of PRP } & \multicolumn{2}{|c|}{ Algorithm of HS } & \multicolumn{2}{|c|}{ New algorithm } \\
\hline & & NOI & NOF & NOI & NOF & NOI & NOF \\
\hline \multirow[t]{7}{*}{ Cubic } & 4 & 15 & 45 & 12 & 35 & 12 & 36 \\
\hline & 10 & 16 & 47 & 13 & 37 & 12 & 36 \\
\hline & 50 & 16 & 47 & 13 & 37 & 12 & 36 \\
\hline & 100 & 16 & 47 & 13 & 37 & 13 & 38 \\
\hline & 500 & 16 & 47 & 13 & 37 & 13 & 38 \\
\hline & 1000 & 16 & 47 & 13 & 37 & 13 & 38 \\
\hline & 5000 & 16 & 47 & 13 & 37 & 13 & 38 \\
\hline Extended & 4 & 7 & 18 & 7 & 18 & 6 & 16 \\
\hline \multirow[t]{6}{*}{ PSC1 } & 10 & 6 & 16 & 6 & 16 & 6 & 16 \\
\hline & 50 & 6 & 16 & 6 & 16 & 6 & 16 \\
\hline & 100 & 7 & 18 & 7 & 18 & 6 & 16 \\
\hline & 500 & 7 & 18 & 7 & 18 & 6 & 16 \\
\hline & 1000 & 7 & 18 & 7 & 18 & 6 & 16 \\
\hline & 5000 & 7 & 18 & 7 & 18 & 6 & 16 \\
\hline \multirow[t]{7}{*}{ GCentral } & 4 & 22 & 159 & 22 & 159 & 21 & 147 \\
\hline & 10 & 22 & 159 & 22 & 159 & 21 & 147 \\
\hline & 50 & 22 & 159 & 22 & 159 & 22 & 159 \\
\hline & 100 & 22 & 159 & 22 & 159 & 22 & 159 \\
\hline & 500 & 23 & 171 & 23 & 171 & 22 & 159 \\
\hline & 1000 & 23 & 171 & 23 & 171 & 22 & 159 \\
\hline & 5000 & 30 & 270 & 28 & 248 & 23 & 173 \\
\hline \multirow[t]{7}{*}{ Miele } & 4 & 37 & 116 & 28 & 85 & 29 & 88 \\
\hline & 10 & 37 & 116 & 31 & 102 & 29 & 88 \\
\hline & 50 & 44 & 148 & 31 & 102 & 35 & 119 \\
\hline & 100 & 44 & 148 & 33 & 114 & 35 & 119 \\
\hline & 500 & 44 & 148 & 40 & 146 & 36 & 121 \\
\hline & 1000 & 50 & 180 & 46 & 176 & 36 & 121 \\
\hline & 5000 & 50 & 180 & 54 & 211 & 43 & 159 \\
\hline \multirow[t]{7}{*}{ OSP } & 4 & 8 & 45 & 8 & 45 & 8 & 44 \\
\hline & 10 & 13 & 57 & 13 & 58 & 13 & 58 \\
\hline & 50 & 37 & 147 & 36 & 133 & 32 & 113 \\
\hline & 100 & 49 & 176 & 49 & 185 & 47 & 166 \\
\hline & 500 & 110 & 341 & 112 & 353 & 109 & 333 \\
\hline & 1000 & 161 & 493 & 156 & 473 & 156 & 480 \\
\hline & 5000 & 276 & 844 & 256 & 774 & 275 & 833 \\
\hline \multirow[t]{7}{*}{ Powell } & 4 & 35 & 87 & 38 & 108 & 29 & 74 \\
\hline & 10 & 35 & 87 & 38 & 108 & 29 & 74 \\
\hline & 50 & 35 & 87 & 38 & 108 & 29 & 74 \\
\hline & 100 & 35 & 87 & 40 & 122 & 29 & 74 \\
\hline & 500 & 35 & 87 & 41 & 124 & 29 & 74 \\
\hline & 1000 & 35 & 87 & 41 & 124 & 32 & 92 \\
\hline & 5000 & 35 & 87 & 41 & 124 & 32 & 92 \\
\hline \multirow[t]{7}{*}{ Wood } & 4 & 29 & 67 & 30 & 68 & 27 & 63 \\
\hline & 10 & 29 & 67 & 30 & 68 & 27 & 63 \\
\hline & 50 & 29 & 67 & 30 & 68 & 28 & 65 \\
\hline & 100 & 30 & 69 & 30 & 68 & 28 & 65 \\
\hline & 500 & 30 & 69 & 30 & 68 & 28 & 65 \\
\hline & 1000 & 30 & 69 & 30 & 68 & 28 & 65 \\
\hline & 5000 & 30 & 69 & 30 & 68 & 28 & 65 \\
\hline Total & & 1734 & 5922 & 1679 & 5856 & 1569 & 5322 \\
\hline
\end{tabular}

Table (2): Comparative Performance of the two Algorithms ( RMIL, $\beta_{k}^{1}$ and New Conjugate Gradient Method)

\begin{tabular}{cccccccc}
\hline Testfunction & $\mathbf{N}$ & \multicolumn{2}{c}{ Algorithm of RMIL } & \multicolumn{2}{c}{$\boldsymbol{\beta}_{\boldsymbol{k}}^{\mathbf{1}}$} & \multicolumn{2}{c}{ New algorithm } \\
\cline { 2 - 7 } Cubic & & NOI & NOF & NOI & NOF & NOI & NOF \\
& 4 & 16 & 47 & 13 & 37 & 12 & 36 \\
& 10 & 16 & 47 & 13 & 37 & 12 & 36 \\
& 50 & 16 & 47 & 13 & 37 & 12 & 36 \\
& 100 & 16 & 47 & 13 & 37 & 13 & 38 \\
& 500 & 16 & 47 & 13 & 37 & 13 & 38 \\
Extended & 1000 & 16 & 47 & 13 & 37 & 13 & 38 \\
PSC1 & 5000 & 16 & 47 & 14 & 39 & 13 & 38 \\
& 4 & 7 & 18 & 7 & 18 & 6 & 16 \\
& 10 & 6 & 16 & 6 & 16 & 6 & 16 \\
& 50 & 6 & 16 & 6 & 16 & 6 & 16 \\
\hline
\end{tabular}




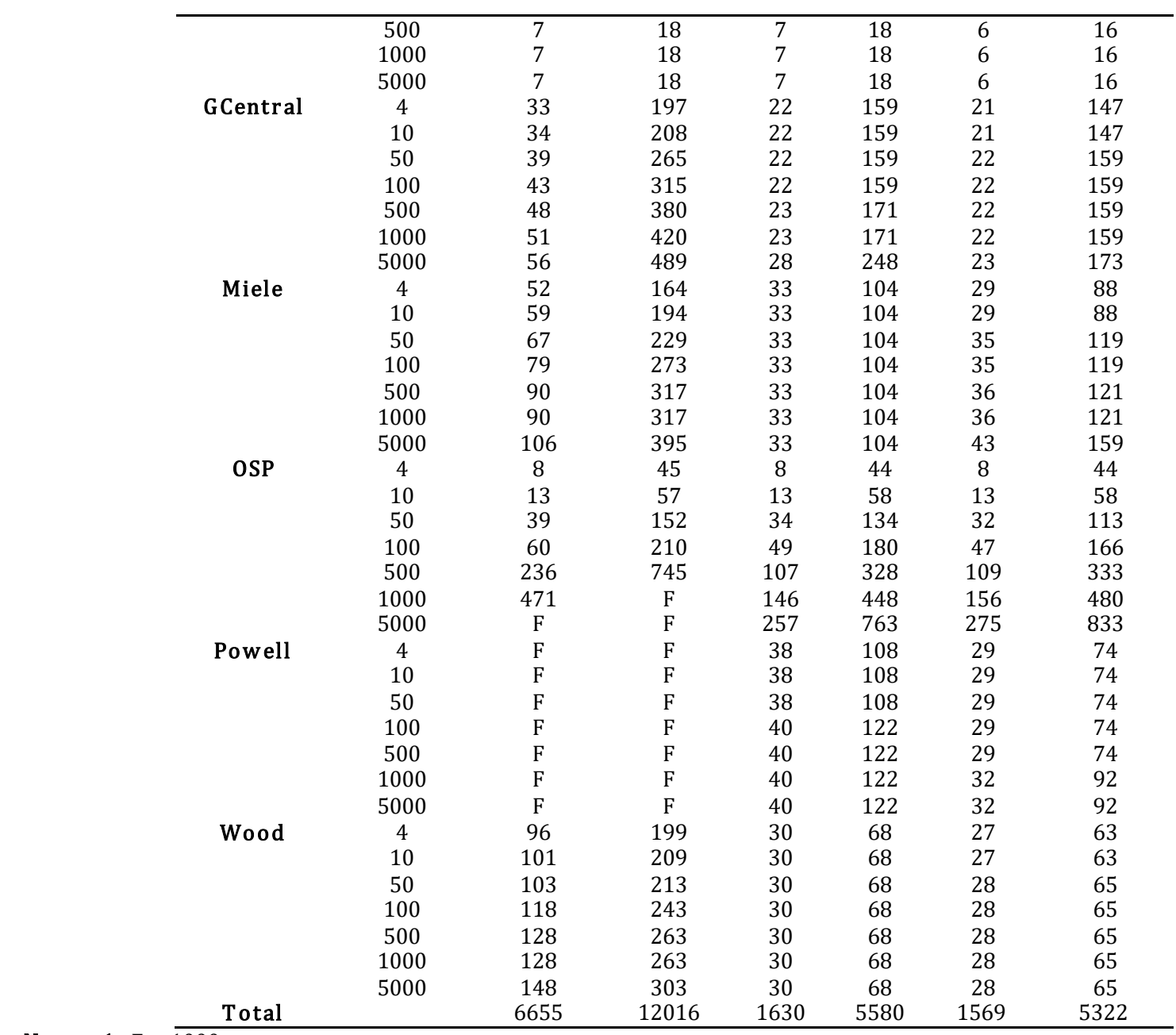

Notes : 1- F $>1000$.

2- We took $\mathrm{F}=500$ for summation.

Table(3): Percentage of Improving of the New Method

\begin{tabular}{ccc}
\hline & Algorithm of PRP & New Algorithm \\
\hline NOI & $100 \%$ & $90.48442907657 \%$ \\
NOF & $100 \%$ & $89.8682877406 \%$ \\
& Algorithm of HS & New Algorithm \\
NOI & $100 \%$ & $93.4484812388 \%$ \\
NOF & $100 \%$ & $90.881147541 \%$ \\
& Algorithm of RMIL & New Algorithm \\
NOI & $100 \%$ & $23.5762584523 \%$ \\
NOF & $100 \%$ & $44.2909454061 \%$ \\
& $\beta_{k}^{1}$ & New Algorithm \\
NOI & $100 \%$ & $96.2576687117 \%$ \\
NOF & $100 \%$ & $95.376344086 \%$ \\
\hline
\end{tabular}

\section{Conclusion}

In this paper, we suggested a new conjugate gradient method for unconstrained optimization. Implemented and tested to some extent, while numerical tes ts were carried out, on low and high dimensionality problems, and comparisons were made amongst different test functions with inexact line search. Some of the num erical resul ts have been reported. 


\section{References:}

[1] E. Polak \& G. Ribiere, Note sur la convergence de directions conjugees, Rev. Francaise Inform Recherche Operationelle, 3(16)(1969), 35-43, https://doi.org/10.1051/m2an/196903r100351

[2] G.H. Yu \& L.T. Guan, Modified PRP Methods with sufficient desent property and their convergence properties. Acta Scientiarum Naturalium Universitatis Sunyatseni(Chinese), 45(4)(2006), 11-14.

[3] J. C. Gilbert \& J. Nocedal, Global convergence properties of conjugate gradient methods for optimization, SIAM J. Optim. 2(1)(1992), 21-42, https://doi.org/10.1137/0802003

[4] J. Liu \& X. Wu, New three-term conjugate gradient method for solving unconstrained optimization problems, Science asia, 40(4)(2014), 295-300, https://doi.org/10.2306/scienceasia1513-1874.2014.40.295

[5] L. Zhang, W. Zhou \& D.H . Li, A descent modified Polak-Ribi`ere-Polyak conjugate gradi ent method and its global convergence, IMA Journal of Numerical Analysis, 26(4)(2006), 629-640, https://doi.org/10.1093/imanum/drl016

[6] L. Zhang, W. Zhou \& D.H. Li, Some descent three-term conjugate gradient methods and their global convergence, Optimization Methods and Software, 22(4)(2007), 697-711, https://doi.org/10.1080/10556780701223293

[7] M. , Al-Baali, Descent property and global convergence of the Fletcher Reeves method with inexact line search, IMA J. Numer. Anal, 5(1)(1985), 121-124, https://doi.org/10.1093/imanum/5.1.121

[8] M.R. Hestenes \& E. Steifel, Method of conjugate gradient for solving linear equations, J.Res. Nat. Bur. Stand, 49(1952), 409-436.

[9] M. Rivaie, M Mamat, W.J. Leong \& M. Ismail, A new class of nonlinear conjugate gradient coefficients with global convergence properties, Applied Mathematics and Computation, 2189(2012), 11323-11332, http://dx.doi.org/10.1016/j.amc.2012.05.030

[10] R., Fletcher, Practical Methods of Optimization vol.1: Unconstrained Optimization. Jhon Wiley \& Sons, New York, 1987.

[11] Y.H. Dai \& Y. Yuan, A nonlinear conjugate gradient with a strong global convergence properties, SIAM J. Optim. 10(1)(1999), 177-182.

[12] Y. Xiao, H. Song \& Z. Wang, A modified conjugate gradient algorithm with cyclic Barzilai-Borwein steplength for unconstrained optimization, Journal of Computational and Applied Mathematics, 236(13)(2012), 3101-3110, https://doi.org/10.1016/j.cam.2012.01.032 